\title{
When is the rate function of a random vector strictly convex?*
}

\author{
Vladislav Vysotsky ${ }^{\dagger}$
}

\begin{abstract}
We give a necessary and sufficient condition for strict convexity of the rate function of a random vector in $\mathbb{R}^{d}$. This condition is always satisfied when the random vector has finite Laplace transform. We also completely describe the effective domain of the rate function under a weaker condition.
\end{abstract}

Keywords: rate function; convex conjugate; Legendre-Fenchel transform; strictly convex; strict convexity; effective domain; steep; essentially smooth; essentially strictly convex. MSC2020 subject classifications: Primary 60E10; 26B25, Secondary 60F10.

Submitted to ECP on September 16, 2020, final version accepted on June 15, 2021. Supersedes arXiv:2009.06809v2.

\section{Introduction}

Let $X$ be a random vector in $\mathbb{R}^{d}$ and $I_{X}$ be its rate function, given by

$$
I_{X}(v):=\sup _{u \in \mathbb{R}^{d}}\left(u \cdot v-\log \mathbb{E} e^{u \cdot X}\right), \quad v \in \mathbb{R}^{d},
$$

where '.' stands for the scalar product in $\mathbb{R}^{d}$. This function is the convex conjugate of the logarithmic Laplace transform of $X$, defined by $K_{X}(u):=\log \mathbb{E} e^{u \cdot X}$ for every $u \in \mathbb{R}^{d}$.

The function $K_{X}$ takes values in $(-\infty,+\infty]$, satisfies $K(0)=0$, and is convex by Hölder's inequality. Then $I_{X}$ is also convex and finite at least at one point ([8, Theorem 12.2]), and it takes values in $[0,+\infty]$. The effective domain $\mathcal{D}\left(I_{X}\right)$ of $I_{X}$, defined by

$$
\mathcal{D}\left(I_{X}\right):=\left\{v \in \mathbb{R}^{d}: I_{X}(v)<+\infty\right\},
$$

is convex and non-empty, and so is the effective domain $\mathcal{D}\left(K_{X}\right)$ of $K_{X}$.

It is easy to show that $K_{X}$ is differentiable at every point of $\operatorname{int} \mathcal{D}\left(K_{X}\right)$ ([2, Corollary 7.1]). When the set $\operatorname{int} \mathcal{D}\left(K_{X}\right)$ is non-empty, we say that $K_{X}$ is steep (at the boundary of its effective domain) if $\lim _{n \rightarrow \infty}\left|\nabla K_{X}\left(u_{n}\right)\right|=\infty$ for every sequence $u_{1}, u_{2}, \ldots$ in int $\mathcal{D}\left(K_{X}\right)$ converging to a point in $\partial \mathcal{D}\left(K_{X}\right)$. Note that $K_{X}$ is steep when it is finite at every point.

The property of steepness appears in a number of general convex-analytic results concerning the so-called essentially smooth convex functions on $\mathbb{R}^{d}$ ([8, Section 26]). In the context of large deviations probabilities this property features in the important

\footnotetext{
*This work was supported in part by Dr Perry James (Jim) Browne Research Centre.

${ }^{\dagger}$ University of Sussex, United Kingdom. E-mail: v.vysotskiy@sussex.ac.uk
} 
When is the rate function of a random vector strictly convex?

Gärtner-Ellis theorem ([4, Section 2.3]). The assumption $0 \in \operatorname{int} \mathcal{D}\left(K_{X}\right)$, which is of course stronger than $\operatorname{int} \mathcal{D}\left(K_{X}\right) \neq \varnothing$, is crucial for classical Cramér's theorem ([7, Section 2.4]), where the rate function $I_{X}$ plays the key role.

Let us recall a few more definitions. For any $A \subset \mathbb{R}^{d}$, denote by conv $A$ (resp. aff $A$ ) the convex hull (resp. affine hull) of $A$, i.e. the minimal convex (resp. affine) subset of $\mathbb{R}^{d}$ containing $A$; denote by $\operatorname{rint} A$ is the relative interior of $A$, i.e. the interior of $A$ in the relative topology of aff $A$; and let $\partial_{\text {rel }} A:=\operatorname{cl} A \backslash \operatorname{rint} A$ be the relative boundary of $A$. Note that $\operatorname{rint} A=A$ if $A$ consists of a single point.

The topological support of a random vector $X$ in $\mathbb{R}^{d}$, denoted by $\operatorname{supp} X$, is the minimal by inclusion closed set $S \subset \mathbb{R}^{d}$ such that $\mathbb{P}(X \in S)=1$. The convex support of $X$ is

$$
C_{X}:=\operatorname{conv}(\operatorname{supp} X) .
$$

A hyperplane $L \subset \mathbb{R}^{d}$ supports a convex set $C \subset \mathbb{R}^{d}$ if $L$ intersects $C$ and $C$ is contained in either of the two half-spaces of $\mathbb{R}^{d}$ that have $L$ as their boundary ( $C \subset L$ is possible).

We say that $I_{X}$ is strictly convex on a set $A \subset \mathcal{D}\left(I_{X}\right)$ if $I_{X}$ is affine on no line segment contained in $A$, and $I_{X}$ is strictly convex if it is strictly convex on $\mathcal{D}\left(I_{X}\right)$.

Our starting point is the following assertion.

Proposition 1.1. Let $X$ be a random vector in $\mathbb{R}^{d}, d \geq 1$.

a) We have

$$
\operatorname{rint} C_{X} \subset \mathcal{D}\left(I_{X}\right) \subset \operatorname{cl} C_{X},
$$

hence $\operatorname{rint} \mathcal{D}\left(I_{X}\right)=\operatorname{rint} C_{X}$. Moreover, $I_{X}=+\infty$ on every hyperplane $L$ in $\mathbb{R}^{d}$ supporting $\operatorname{cl} C_{X}$ and such that $\mathbb{P}(X \in L)=0$.

b) $I_{X}$ is strictly convex on $\operatorname{rint} \mathcal{D}\left(I_{X}\right)$ if and only if int $\mathcal{D}\left(K_{X}\right) \neq \varnothing$ and $K_{X}$ is steep.

We will prove this result in full for completeness of exposition. The inclusions in (1.1) are not new but we do not have exact references. They are stated in [2, Theorem 9.1], which however concerns only a specific type of distributions. They follow from [3, Theorems 2.1 2.3, 3.2] but do not appear in [3] explicitly. The last claim of Part a) is in [2, Theorem 9.5]. Part b) states a particular case of a general convex-analytic result [8, Theorem 26.3], with the novelty that we strengthened the direct implication.

The main result of this note is a necessary and sufficient condition (see Theorem 2.11) for strict convexity of $I_{X}$ on its whole effective domain $\mathcal{D}\left(I_{X}\right)$. This condition is always satisfied when the Laplace transform of $X$ is finite in the whole of $\mathbb{R}^{d}$, and thus $I_{X}$ is strictly convex for such $X$.

In view of Proposition 1.1, we only need to characterize strict convexity of the rate function on the relative boundary of $\mathcal{D}\left(I_{X}\right)$. Our approach is based on the following result.

Theorem 1.2. Let $X$ be a random vector in $\mathbb{R}^{d}, d \geq 1$, and $L$ be a hyperplane in $\mathbb{R}^{d}$ supporting $C_{X}$ and such that $0<\mathbb{P}(X \in L)<1$. Then

$$
I_{X}(v)=I_{X \mid L}(v)-\log \mathbb{P}(X \in L), \quad v \in L,
$$

if and only if

$$
\operatorname{Pr}_{L}\left(\operatorname{rint} \mathcal{D}\left(K_{X}\right)\right)=\operatorname{Pr}_{L}\left(\operatorname{rint} \mathcal{D}\left(K_{X \mid L}\right)\right),
$$

where $X \mid L$ is a random vector distributed as $X$ conditioned to be in $L$ and $\operatorname{Pr}_{L}$ denotes the orthogonal projection from $\mathbb{R}^{d}$ onto $L$. 
When is the rate function of a random vector strictly convex?

Assume that condition (1.3) is satisfied for every hyperplane $L$ as above. Then we can apply Proposition 1.1 to each of the $I_{X \mid L}$ 's. By (1.2), this ensures that $\operatorname{rint} \mathcal{D}\left(C_{X \mid L}\right) \subset$ $\mathcal{D}\left(I_{X}\right)$, and $I_{X}$ is strictly convex on every set rint $\mathcal{D}\left(C_{X \mid L}\right)$ if and only if the $K_{X \mid L}$ 's are steep. The main idea is to apply this argument to the $X \mid L^{\prime}$ s and further on recursively, using that the sets $\operatorname{rint} \mathcal{D}\left(I_{X \mid L}\right)$ are disjoint with rint $\mathcal{D}\left(I_{X}\right)$ by $\operatorname{dim} C_{X \mid L}<\operatorname{dim} C_{X}$. Under appropriate conditions, which ensure that (1.3) is satisfied at every step of the recursion, this lets us fully describe $\mathcal{D}\left(I_{X}\right)$ (see Corollary 2.13) and characterize strict convexity of $I_{X}$ (see Theorem 2.11). We give the details in the next section, where we also comment on condition (1.3) (see Remark 2.6).

The property of strict convexity can be useful when proving uniqueness of solutions to minimization problems involving $I_{X}$. Such problems arise from large deviations principles, most naturally in Cramér's theorem (see [7, Section 2.4]) on random walks in $\mathbb{R}^{d}$. There are functional versions of this result, which describe scaled trajectories of random walks and continuous time analogues for Lévy processes (see [4, Section 5.1 and 5.2] and [6]). In both cases, if the increments have finite Laplace transform, then the large deviations are described by the rate function $I$ of the form $I(f)=\int_{0}^{1} I_{X}\left(f^{\prime}(t)\right) d t$ for $f$ in $A C_{0}$, the space of coordinate-wise absolutely continuous $\mathbb{R}^{d}$-valued functions on $[0,1]$ such that $f(0)=0$.

For concrete examples, let $\left(S_{n}\right)_{n \in \mathbb{N}}$ be a random walk with i.i.d. increments distributed as $X$. When $I_{X}$ is strictly convex, its unique minimizer $b$ over a convex Borel set $B \subset \mathbb{R}^{d}$ that meets $\mathcal{D}\left(I_{X}\right)$ can be interpreted as the limit constant in the law of large numbers for the averages $S_{n} / n$ conditioned to be in $B$. Under this conditioning, a typical trajectory $\left(S_{k} / n\right)_{1 \leq k \leq n}$ of the random walk with $\mathcal{D}\left(K_{X}\right)=\mathbb{R}^{d}$ is asymptotically linear with slope $b$ because the function $f_{0}(t)=b t$ is the unique minimizer of $I$ over the set $\left\{f \in A C_{0}: f(1) \in B\right\}$. This follows from Jensen's inequality using that $I_{X}$ is strictly convex (by Corollary 2.12). When $0 \in \operatorname{int} \mathcal{D}\left(K_{X}\right)$ but $\mathcal{D}\left(K_{X}\right) \neq \mathbb{R}^{d}$, the rate function $I$ has a more complicated form, and without strict convexity of $I_{X}$ the argument above becomes less simple (see [5, pp. 16-17]). More elaborate examples arise, e.g. in the study [1] of large deviations of the perimeter and the area of convex hulls of planar random walks, where strict convexity of $I_{X}$ simplified considerations.

Finally, we note that relating the conditional limit laws to the minimizers of the rate function, as above, corresponds to the fundamental Gibbs conditioning principle of statistical mechanics (see [4], including Sections 3.3 and 7.3).

\section{Main result}

We first recall some facts on the structure of convex sets.

A face of a non-empty convex set $C \subset \mathbb{R}^{d}$ is a convex subset $C^{\prime}$ of $C$ such that every closed line segment in $C$ with a relative interior point in $C^{\prime}$ has both endpoints in $C^{\prime}$. Note that $C$ itself is a face; the zero-dimensional faces are called the extreme points of $C$. If $L \subset \mathbb{R}^{d}$ is a hyperplane supporting $C$, then $C \cap L$ is face of $C$. Every face of such form is called exposed.

Denote by $\mathcal{F}(C)$ the set of non-empty faces of $C$ and by $\mathcal{F}^{*}(C)$ its subset of maximal proper faces, defined by

$$
\mathcal{F}^{*}(C):=\left\{C^{\prime} \in \mathcal{F}(C) \backslash\{C\}: C^{\prime} \not \subset C^{\prime \prime} \text { for every } C^{\prime \prime} \in \mathcal{F}(C) \backslash\left\{C, C^{\prime}\right\}\right\} .
$$

We will use extensively that every face in $\mathcal{F}^{*}(C)$ is exposed (this follows from [8, Theorem 11.6 and Corollary 18.1.3]). Our need in the set $\mathcal{F}^{*}(C)$ is due to the following result. 
When is the rate function of a random vector strictly convex?

Lemma 2.1. Let $C \subset \mathbb{R}^{n}$ be a non-empty convex set. Then

$$
C \backslash \operatorname{rint} C=\bigcup_{C^{\prime} \in \mathcal{F}^{*}(C)} C^{\prime} .
$$

Proof. In fact, the set $C \backslash \operatorname{rint} C$ contains every proper face of $C$ by [8, Corollary 18.1.3]. On the other hand, by [8, Theorem 11.6], for every point in $C \backslash \operatorname{rint} C$ there is a hyperplane $L$ containing this point and supporting $C$ but not containing $C$. Then $C \cap L$ is a proper face of $C$. To finish the proof it remains to argue that for any proper face $C^{\prime}$ of $C$ is contained in a maximal proper face of $C$.

Let us use induction in $\operatorname{dim} C^{\prime}$. In the base case $\operatorname{dim} C^{\prime}=\operatorname{dim} C-1$, we always have $C^{\prime} \in \mathcal{F}^{*}(C)$. Indeed, if this were not true, there would be a proper face $C^{\prime \prime}$ of $C$ other than $C^{\prime}$ that strictly contains $C^{\prime}$. Then $C^{\prime}$ would be a face of $C^{\prime \prime}$ (by definition of a face), hence $\operatorname{dim} C^{\prime}<\operatorname{dim} C^{\prime \prime}$ by [8, Corollary 18.1.3]. This is a contradiction because there are no faces of $C$ other than itself of dimension $\operatorname{dim} C$.

Let us prove the inductive step. If $C^{\prime}$ is maximal, we are done. Otherwise, choose $C^{\prime \prime}$ as above. By the assumption of induction, there is a $C^{\prime \prime \prime} \in \mathcal{F}^{*}(C)$ containing $C^{\prime \prime}$. This is a face required.

We now consider faces of the convex support $C_{X}$ of a random vector $X$ in $\mathbb{R}^{d}$. First note that $C_{X}$ is not necessarily closed; it can be even open.

Example 2.2. Let $X$ be a random vector in $\mathbb{R}^{2}$ such that $\operatorname{supp} X=\left\{(x, y) \in \mathbb{R}^{2}: y \geq\right.$ $\left.\frac{1}{1+x^{2}}\right\}$. Then $C_{X}$ is the open upper half-plane.

However, we have the following measurability result.

Lemma 2.3. Let $X$ be a random vector in $\mathbb{R}^{d}, d \geq 1$. Then $C_{X}$ is a Borel subset of $\mathbb{R}^{d}$, and so is every $C \in \mathcal{F}^{*}\left(C_{X}\right)$.

Proof. By Carathéodory's theorem ([8, Theorem 17.1]), every point in $C_{X}$ is a convex combination of $d+1$ points in $\operatorname{supp} X$. Then $C_{X}=\cup_{n=1}^{\infty} \operatorname{conv}\left(\operatorname{supp} X \cap\left\{u \in \mathbb{R}^{d}:\|u\| \leq n\right\}\right)$. By [8, Theorem 17.2], each set under the union is closed, and hence $C_{X}$ is Borel.

Every $C \in \mathcal{F}^{*}\left(C_{X}\right)$ is an exposed face of $C_{X}$, therefore $C=C_{X} \cap L$ for some affine hyperplane $L$ supporting $C_{X}$. Hence $C$ also is a Borel set.

The lemma ensures that the following set is well-defined:

$$
\mathcal{F}_{+}^{*}\left(C_{X}\right):=\left\{C \in \mathcal{F}^{*}\left(C_{X}\right): \mathbb{P}(X \in C)>0\right\} .
$$

In the results below it is useful to know when this set is empty. We give the following criterion.

Lemma 2.4. Let $X$ be a random vector in $\mathbb{R}^{d}, d \geq 1$. Then $\mathcal{F}_{+}^{*}\left(C_{X}\right)$ is empty if and only if there is no hyperplane $L$ in $\mathbb{R}^{d}$ supporting $C_{X}$ and such that $0<\mathbb{P}(X \in L)<1$.

Proof. If $L$ is a hyperplane supporting $C_{X}$, then either $C_{X} \subset L$, in which case $\mathbb{P}(X \in$ $L)=1$, or $C_{X} \cap L \in \mathcal{F}^{*}\left(C_{X}\right)$, hence from $\mathcal{F}_{+}^{*}\left(C_{X}\right)=\varnothing$ we get $\mathbb{P}\left(X \in C_{X} \cap L\right)=0$ and thus $\mathbb{P}(X \in L)=0$. This proves the direct implication.

To prove the reverse implication, assume that there is a $C \in \mathcal{F}_{+}^{*}\left(C_{X}\right)$. This is an exposed face of $C_{X}$, therefore $C=C_{X} \cap L$ for some affine hyperplane $L$ supporting $C_{X}$. Then $\mathbb{P}(X \in L)=\mathbb{P}(X \in C)>0$, hence by the assumption, it must be $\mathbb{P}(X \in L)=1$. Hence $\operatorname{supp} X \subset L$ (because $L$ is a closed set) and therefore $C_{X} \subset L$. Thus, $C$ is not a proper face of $C_{X}$, which is a contradiction.

For every random vector $X$ in $\mathbb{R}^{d}$ and $C \in \mathcal{F}_{+}^{*}\left(C_{X}\right)$, let $X \mid C$ be a random vector distributed as $X$ conditioned on $X \in C$.

We now give two key definitions, both having recursive structure. 
When is the rate function of a random vector strictly convex?

Definition 2.5. We say that $K_{X}$ has the projection property ${ }^{1}$ if

a) for every hyperplane $L$ in $\mathbb{R}^{d}$ supporting $C_{X}$ and such that $0<\mathbb{P}(X \in L)<1$, we have

$$
\operatorname{Pr}_{L}\left(\operatorname{rint} \mathcal{D}\left(K_{X}\right)\right)=\operatorname{Pr}_{L}\left(\operatorname{rint} \mathcal{D}\left(K_{X \mid L}\right)\right) ;
$$

b) $K_{X \mid C}$ has the projection property for every $C \in \mathcal{F}_{+}^{*}\left(C_{X}\right)$.

The projection property is well-defined since the definition allows us to identify, using recursion in $\operatorname{dim} C_{X}$, whether each particular $K_{X}$ has this property nor not. This is true because 1) $\operatorname{dim} C_{X \mid C} \leq \operatorname{dim} C<\operatorname{dim} C_{X}$ for every $\left.C \in \mathcal{F}_{+}^{*}\left(C_{X}\right) ; 2\right)$ the recursion terminates (confirming that $K_{X}$ has the property) if Conditions a) and b) hold vacuously, namely when $\mathcal{F}_{+}^{*}\left(C_{X}\right)=\varnothing$ (by Lemma 2.4); and 3) the recursion always terminates since $\mathcal{F}_{+}^{*}\left(C_{X}\right)=\varnothing$ when $\operatorname{dim} C_{X}=0$, i.e. $X$ is constant a.s.

Remark 2.6. Let us comment on Condition a).

a) Each set $\mathcal{D}\left(K_{X \mid L}\right)$ is a right cylinder. So is its relative interior, which satisfies

$$
\operatorname{Pr}_{L}\left(\operatorname{rint} \mathcal{D}\left(K_{X \mid L}\right)\right)=\operatorname{rint}\left(\operatorname{Pr}_{L} \mathcal{D}\left(K_{X \mid L}\right)\right)=\operatorname{rint}\left(L \cap \mathcal{D}\left(K_{X \mid L}\right)\right)=L \cap \operatorname{rint} \mathcal{D}\left(K_{X \mid L}\right),
$$

where the first and the last equalities follow from [8, Theorem 6.6 and Corollary 6.5.1].

b) We always have $\mathcal{D}\left(K_{X}\right) \subset \mathcal{D}\left(K_{X \mid L}\right)$. This follows from

$$
K_{X}(u) \geq \log \mathbb{E}\left(e^{u \cdot X} \mathbb{1}_{\{X \in L\}}\right)=K_{X \mid L}(u)+\log \mathbb{P}(X \in L), \quad u \in \mathbb{R}^{d} .
$$

Assume additionally that $\mathcal{D}\left(K_{X}\right)$ is not entirely contained in the relative boundary of $\mathcal{D}\left(K_{X \mid L}\right)$; this holds, e.g. when $\operatorname{int} \mathcal{D}\left(K_{X}\right) \neq \varnothing$ or $0 \in \operatorname{rint} \mathcal{D}\left(K_{X \mid L}\right)$. Then

$$
\operatorname{Pr}_{L}\left(\operatorname{rint} \mathcal{D}\left(K_{X}\right)\right) \subset \operatorname{Pr}_{L}\left(\operatorname{rint} \mathcal{D}\left(K_{X \mid L}\right)\right)
$$

because rint $\mathcal{D}\left(K_{X}\right) \subset \operatorname{rint} \mathcal{D}\left(K_{X \mid L}\right)$ by [8, Corollary 6.5.2]. Thus, (1.3) means that the projection of rint $\mathcal{D}\left(K_{X}\right)$ on $L$ does not increase if $X$ is replaced by $X \mid L$.

c) Every supporting hyperplane $L$ to $C_{X}$ is of the form $L=\left\{v \in \mathbb{R}^{d}: \ell \cdot v=h_{C_{X}}(\ell)\right\}$, where $\ell \in \mathbb{S}^{d-1}$ is a unit vector orthogonal to $L$ and $h_{C_{X}}$ is the support function of $C_{X}$ defined by $h_{C_{X}}(u):=\sup _{v \in C_{X}} u \cdot v, u \in \mathbb{R}^{d}$. Since $\ell \in \mathcal{D}\left(h_{C_{X}}\right)$ if and only if $C_{X}$ is bounded in direction $\ell$ (equivalently, $\operatorname{supp} X$ is bounded in direction $\ell$ ), this implies that $\{a \ell: a \geq 0\} \subset \mathcal{D}\left(K_{X}\right)$. It is therefore easy to see that $\operatorname{Pr}_{L}\left(\operatorname{rint} \mathcal{D}\left(K_{X}\right)\right)=L$ when $\ell \in \operatorname{int} \mathcal{D}\left(h_{C_{X}}\right)$. Hence for such $\ell$ equality (1.3) always holds true by $\mathcal{D}\left(K_{X}\right) \subset$ $\mathcal{D}\left(K_{X \mid L}\right)$.

Thus, it suffices to check the assumption of Condition a) only for hyperplanes supporting $C_{X}$ that are orthogonal to directions in the set $\partial \mathcal{D}\left(h_{C_{X}}\right) \cap \mathbb{S}^{d-1}$. For $d=2$ this set contains at most two directions because $\mathcal{D}\left(h_{C_{X}}\right)$ is a convex cone.

We now give a few examples.

Example 2.7. $K_{X}$ has the projection property in the following cases:

a) $\mathcal{F}_{+}^{*}\left(C_{X}\right)$ is empty. In particular, this holds true when $\mathbb{P}\left(X \in \partial_{\text {rel }} C_{X}\right)=0$; see (2.1).

b) $\mathcal{D}\left(K_{X}\right)=\mathbb{R}^{d}$ or, equivalently, $\mathbb{E} e^{u \cdot X}<\infty$ for every $u \in \mathbb{R}^{d}$; cf. (2.2).

c) $d=1$.

\footnotetext{
${ }^{1}$ Strictly speaking, this is a property of the distribution of $X$ rather than of $K_{X}$. However, the distributions that satisfy $\operatorname{int} \mathcal{D}\left(K_{X}\right) \neq \varnothing$ are determined by their Laplace transform (this reduces to $d=1$, where Theorem 6a in Chapter VI of [9] applies). Note that our main result, Theorem 2.11.b, assumes int $\mathcal{D}\left(K_{X}\right) \neq \varnothing$.
} 
d) $d=2$ and equality (1.3) holds true for every line $L$ of the form $L=$ aff $C$, where $C \in \mathcal{F}_{+}^{*}\left(C_{X}\right)$ is unbounded (there are at most two such faces).

Indeed, such lines are orthogonal to the directions in $\partial \mathcal{D}\left(h_{C_{X}}\right) \cap \mathbb{S}^{1}$ and then $\operatorname{Re}$ mark 2.6.c applies. Clearly, Condition a) in Definition 2.5 is satisfied by Example 2.7.c since $\operatorname{dim} C_{X \mid C} \leq 1$ for every $C \in \mathcal{F}_{+}^{*}\left(C_{X}\right)$.

Our second key definition is as follows.

Definition 2.8. If $\mathcal{D}\left(K_{X}\right)$ has non-empty interior, we say that $K_{X}$ is totally steep if $K_{X}$ is steep and $K_{X \mid C}$ is totally steep for every $C \in \mathcal{F}_{+}^{*}\left(C_{X}\right)$.

Again, this property is well-defined by recursion in $\operatorname{dim} C_{X}$ because 1) $\operatorname{dim} C_{X \mid C}<$ $\operatorname{dim} C_{X}$ for every $\left.C \in \mathcal{F}_{+}^{*}\left(C_{X}\right) ; 2\right)$ int $\mathcal{D}\left(K_{X \mid C}\right) \neq \varnothing$ for $C \in \mathcal{F}_{+}^{*}\left(C_{X}\right)$ by $\mathcal{D}\left(K_{X}\right) \subset \mathcal{D}\left(K_{X \mid C}\right)$ (cf. (2.2)); 3) $K_{X}$ is totally steep when it is steep and $\mathcal{F}_{+}^{*}\left(C_{X}\right)=\varnothing$; and 4 ) $K_{X}$ is totally steep when $\operatorname{dim} C_{X}=0$.

Example 2.9. $K_{X}$ is totally steep if $\mathcal{D}\left(K_{X}\right)=\mathbb{R}^{d}$.

Example 2.10. Let us construct $K_{X}$ which neither has the projection property nor is totally steep. Put $X:=\left(\alpha X_{1}, \alpha X_{2}+(1-\alpha) X_{3}\right)$, where $X_{1}, X_{2}, X_{3}, \alpha$ are independent non-negative random variables such that $X_{1}$ and $X_{2}$ have the standard exponential distribution with density $e^{-x}$ for $x>0, X_{3}$ has the absolutely continuous distribution with density proportional to $e^{-2 x} /\left(1+x^{3}\right)$ for $x>0$, and $\mathbb{P}(\alpha=0)=\mathbb{P}(\alpha=1)=1 / 2$.

We have $\mathcal{D}\left(K_{X_{3}}\right)=(-\infty, 2]$ and it is easy to check that $K_{X_{3}}^{\prime}(2-)<+\infty$, hence $K_{X_{3}}$ is not steep; and $K_{X_{1}}$ is steep. Furthermore, $K_{X}\left(u_{1}, u_{2}\right)=\frac{1}{2} K_{X_{1}}\left(u_{1}\right)+\frac{1}{2} K_{X_{2}}\left(u_{2}\right)+\frac{1}{2} K_{X_{3}}\left(u_{2}\right)$ for $u_{1}, u_{2} \in \mathbb{R}$; the set $C_{X}$ is the closed positive quadrant in the plane; and $\mathcal{F}_{+}^{*}\left(C_{X}\right)=\{C\}$ with $C:=\{0\} \times[0, \infty)$. We can see that $K_{X}$ is steep but not totally steep because $\mathcal{D}\left(K_{X}\right)=(-\infty, 1) \times(-\infty, 1)$ but for the ordinate line $L=$ aff $C$ supporting $C_{X}$, the random vector $X \mid L$ is distributed as $\left(0, X_{3}\right)$ and thus $K_{X \mid L}$ is not steep. This also shows that Condition a) in Definition 2.5 is violated because $\operatorname{Pr}_{L}\left(\operatorname{rint} \mathcal{D}\left(K_{X \mid L}\right)\right)=\{0\} \times(-\infty, 2)$ but $\operatorname{Pr}_{L}\left(\operatorname{rint} \mathcal{D}\left(K_{X}\right)\right)=\{0\} \times(-\infty, 1)$, and thus $K$ does not have the projection property.

We are now ready to state the main result of the paper.

Theorem 2.11. Let $X$ be a random vector in $\mathbb{R}^{d}, d \geq 1$.

a) If $K_{X}$ satisfies Condition a) in Definition 2.5 of the projection property, then

$$
\mathcal{F}^{*}\left(\mathcal{D}\left(I_{X}\right)\right) \subset\left\{\mathcal{D}\left(I_{X \mid C}\right): C \in \mathcal{F}_{+}^{*}\left(C_{X}\right)\right\} \subset \mathcal{F}\left(\mathcal{D}\left(I_{X}\right)\right) \backslash\left\{\mathcal{D}\left(I_{X}\right)\right\}
$$

b) $I_{X}$ is strictly convex if and only if $\operatorname{int} \mathcal{D}\left(K_{X}\right) \neq \varnothing, K_{X}$ has the projection property, and $K_{X}$ is totally steep.

Let us present a few corollaries.

Corollary 2.12. If $\mathbb{E} e^{u \cdot X}<\infty$ for every $u \in \mathbb{R}^{d}$, then $I_{X}$ is strictly convex.

Proof. This follows directly from Part b) using Examples 2.7.b and 2.9.

Corollary 2.13. If $K_{X}$ has the projection property, then $\mathcal{D}\left(I_{X}\right) \subset C_{X}$ and

$$
\begin{aligned}
& \mathcal{D}\left(I_{X}\right) \\
& =\operatorname{rint} C_{X} \cup \bigcup_{C_{1} \in \mathcal{F}_{+}^{*}\left(C_{X}\right)} \operatorname{rint} C_{X \mid C_{1}} \cup \bigcup_{C_{2} \in \mathcal{F}_{+}^{*}\left(C_{X \mid C_{1}}\right)} \operatorname{rint} C_{X \mid C_{2}} \cup \ldots \cup \bigcup_{C_{d} \in \mathcal{F}_{+}^{*}\left(C_{X \mid C_{d-1}}\right)} \operatorname{rint} C_{X \mid C_{d}} .
\end{aligned}
$$

Proof. We have

$$
\mathcal{D}\left(I_{X}\right)=\operatorname{rint} \mathcal{D}\left(I_{X}\right) \cup \bigcup_{C \in \mathcal{F}^{*}\left(\mathcal{D}\left(I_{X}\right)\right)} C=\operatorname{rint} C_{X} \cup \bigcup_{C_{1} \in \mathcal{F}_{+}^{*}\left(C_{X}\right)} \mathcal{D}\left(I_{X \mid C_{1}}\right),
$$


When is the rate function of a random vector strictly convex?

where the first equality follows from (2.1) and the second one follows from (2.4) and the fact that $\operatorname{rint} \mathcal{D}\left(I_{X}\right)=\operatorname{rint} C_{X}$ (see Proposition 1.1.a). Then we establish the equality claimed by simple induction in $\operatorname{dim} C_{X}$ using that each random vector $\left(X \mid C_{1}\right) \mid C_{2}$ has the same distribution as $X \mid C_{2}$. In the base case $\operatorname{dim} C_{X}=0$ the claim holds by $\mathcal{D}\left(I_{X}\right)=$ $C_{X}=\operatorname{rint} C_{X}$ and $\mathcal{F}_{+}^{*}\left(C_{X}\right)=\varnothing$. The same inductive argument establishes the inclusion $\mathcal{D}\left(I_{X}\right) \subset C_{X}$.

Corollary 2.14. Assume that $K_{X}$ has the projection property. Then $v$ is an extreme point of $\mathcal{D}\left(I_{X}\right)$ if and only if $v$ is an extreme point of $C_{X}$ and $\mathbb{P}(X=v)>0$. For such $v$, we have $I_{X}(v)=-\log \mathbb{P}(X=v)$.

Proof. Assume that $v$ is an extreme point of $C_{X}$ and $\mathbb{P}(X=v)>0$. We use induction in $\operatorname{dim} C_{X}$. In the base case $\operatorname{dim} C_{X}=0$, we simply have $I_{X}(v)=0=-\log (X=v)$. To prove the induction step for $\operatorname{dim} C_{X} \geq 1$, use that by (2.1) there is a face $C \in \mathcal{F}_{+}^{*}\left(C_{X}\right)$ that contains $v$. Then $v$ is an extreme point of $C_{X \mid C}$ because $v \in C_{X \mid C}$ by $\mathbb{P}((X \mid C)=v)>0$ and $v$ is an extreme point of the convex set $C_{X}$ which contains $C_{X \mid C}$.

Since $C$ an exposed face of $C_{X}$, there is a hyperplane $L$ supporting $C_{X}$ such that $C=C_{X} \cap L$. Then $X \mid C$ and $X \mid L$ have the same distribution since $\mathbb{P}(X \in L \backslash C)=0$, and by (1.2) and the assumption of induction we get

$$
I_{X}(v)=I_{X \mid C}(v)-\log \mathbb{P}(X \in C)=-\log \mathbb{P}((X \mid C)=v)-\log \mathbb{P}(X \in C)=-\log \mathbb{P}(X=v) .
$$

Then $I_{X}(v)<\infty$, and thus $v \in \mathcal{D}\left(I_{X}\right)$. Hence $v$ is an extreme point of $\mathcal{D}\left(I_{X}\right)$ because $v$ is an extreme point of the convex set $C_{X}$ which contains $\mathcal{D}\left(I_{X}\right)$ by Corollary 2.13.

Proving the reverse implication is similar. For the induction step, for $\operatorname{dim} \mathcal{D}\left(I_{X}\right) \geq 1$, use that by (2.1) there is a face $F \in \mathcal{F}^{*}\left(\mathcal{D}\left(I_{X}\right)\right)$ that contains $v$. Then $v$ is an extreme point of $F$. By (2.4), $F=\mathcal{D}\left(I_{X \mid C}\right)$ for some face $C \in \mathcal{F}_{+}^{*}\left(C_{X}\right)$, and we can apply the assumption of induction as above.

\section{Proofs}

Proof of Proposition 1.1. a) Recall that $C_{X}=\operatorname{conv}(\operatorname{supp} X)$. Fix a $v \notin \operatorname{cl} C_{X}$. By [8, Corollary 11.5.1], there exists a non-zero $u_{0} \in \mathbb{R}^{d}$ such that $u_{0} \cdot x<u_{0} \cdot v$ for any $x \in \operatorname{supp} X$. In other words, $u_{0} \cdot X<u_{0} \cdot v$ a.s. By the monotone convergence theorem, we get

$$
I(v)=\sup _{u \in \mathbb{R}^{d}}\left(u \cdot v-K_{X}(u)\right) \geq \sup _{a>0}\left(a u_{0} \cdot v-K_{X}\left(a u_{0}\right)\right)=-\inf _{a>0}\left(\log \mathbb{E} e^{a\left(u_{0} \cdot X-u_{0} \cdot v\right)}\right)=+\infty .
$$

Thus, $\mathcal{D}\left(I_{X}\right) \subset \operatorname{cl} C_{X}$.

Furthermore, if $L$ a hyperplane supporting $\operatorname{cl} C_{X}$, take any non-zero $u_{0} \in \mathbb{R}^{d}$ orthogonal to $L$ and directed such that $u_{0} \cdot x \leq u_{0} \cdot v$ for any $x \in \operatorname{supp} X$ and $v \in L$, that is $u_{0} \cdot X \leq u_{0} \cdot v$ a.s. This inequality is strict if $\mathbb{P}(X \in L)=0$, in which case $I(v)=+\infty$ holds true by (3.1), as required.

We now show that $\operatorname{rint} C_{X} \subset \mathcal{D}\left(I_{X}\right)$. Assume that this does not hold. Then, since $\mathcal{D}\left(I_{X}\right) \subset \operatorname{cl} C_{X}$ and the sets $C_{X}$ and $\mathcal{D}\left(I_{X}\right)$ are convex, we have $\operatorname{cl} \mathcal{D}\left(I_{X}\right) \neq \operatorname{cl} C_{X}$ by [8, Corollary 6.3.1]. Therefore, there exists an open ball $B \subset \mathbb{R}^{d}$ such that $\operatorname{cl} \mathcal{D}\left(I_{X}\right) \cap \operatorname{cl} B=\varnothing$ and $\operatorname{cl} C_{X} \cap B \neq \varnothing$.

For any $n \in \mathbb{N}$, let $S_{n}$ be the sum of $n$ independent identically distributed copies of $X$. Then for any $u \in \mathcal{D}\left(K_{X}\right)$, we have

$$
\mathbb{P}\left(S_{n} / n \in B\right)=\mathbb{E}\left[\mathbb{1}\left(S_{n} / n \in B\right)\right] \leq \mathbb{E}\left[\mathbb{1}\left(u \cdot S_{n} \geq n \inf _{v \in \operatorname{cl} B} u \cdot v\right)\right] \leq e^{-n \inf _{v \in \operatorname{cl} B} u \cdot v} \mathbb{E} e^{u \cdot S_{n}},
$$


When is the rate function of a random vector strictly convex?

where the last equality follows from Markov's inequality. Then

$n^{-1} \log \mathbb{P}\left(S_{n} / n \in B\right) \leq \inf _{u \in \mathcal{D}\left(K_{X}\right)}\left(-\inf _{v \in \operatorname{cl} B}\left(u \cdot v-K_{X}(u)\right)\right)=-\sup _{u \in \mathcal{D}\left(K_{X}\right)} \inf _{v \in \operatorname{cl} B}\left(u \cdot v-K_{X}(u)\right)$.

Finally, let us interchange the supremum and the infimum using a minimax result [8, Corollary 37.3.2] on concave-convex functions. This gives

$$
n^{-1} \log \mathbb{P}\left(S_{n} / n \in B\right) \leq-\inf _{v \in \operatorname{cl} B} I_{X}(v) .
$$

This inequality appears, e.g., in [7, Eq. (2.16)].

On the other hand, since $\operatorname{cl} C_{X} \cap B \neq \varnothing, B$ is open, and $C_{X}$ is convex, it follows from [8, Corollary 6.3.2] that rint $C_{X}$ intersects with $B$. Hence, by Carathéodory's theorem ([8, Theorem 17.1]), there is a convex combination $\sum_{i=1}^{m} \alpha_{i} x_{i} \in B$, where $m$ is a positive integer, $x_{i} \in \operatorname{supp} X$ and $\alpha_{i}>0$ for every $1 \leq i \leq m$, and $\sum_{i=1}^{m} \alpha_{i}=1$. By finding a rational approximation to all but one of the $\alpha_{i}$ 's, we get $\frac{1}{n} \sum_{i=1}^{m} n_{i} x_{i} \in B$ for some positive integer $n_{i}$ and $n=\sum_{i=1}^{m} n_{i}$. Furthermore, there exist open balls $B_{i} \subset \mathbb{R}^{d}$ such that $x_{i} \in B_{i}$ for every $1 \leq i \leq m$ and $\frac{1}{n} \sum_{i=1}^{m} n_{i} B_{i} \subset B$. Since each open ball $B_{i}$ intersects with $\operatorname{supp} X$, we have $\mathbb{P}\left(X \in B_{i}\right)>0$. Therefore,

$$
\mathbb{P}\left(S_{n} / n \in B\right) \geq \prod_{i=1}^{m} \mathbb{P}\left(X \in B_{i}\right)^{n_{i}}>0 .
$$

Inequalities (3.2) and (3.3) imply that $\operatorname{cl} B \cap \mathcal{D}\left(I_{X}\right) \neq \varnothing$, which is contradiction. Thus, we proved that $\operatorname{rint} C_{X} \subset \mathcal{D}\left(I_{X}\right) \subset \operatorname{cl} C_{X}$, establishing (1.1). Finally, by [8, Corollary 6.3.1] this gives $\operatorname{rint} C_{X}=\operatorname{rint} \mathcal{D}\left(I_{X}\right)$, as required.

b) Put $d^{\prime}:=\operatorname{dim} C_{X}$. We assume that $d^{\prime} \geq 1$, otherwise the claim is trivial.

Recall that $I_{X}$ is subdifferentiable at a point $v_{0} \in \mathbb{R}^{d}$ if there is a $u \in \mathbb{R}^{d}$ such that the inequality $I_{X}(v) \geq I_{X}\left(v_{0}\right)+u \cdot\left(v-v_{0}\right)$ holds for every $v \in \mathbb{R}^{d}$. We claim that $I_{X}$ is subdifferentiable at no point outside of $\operatorname{rint} \mathcal{D}\left(I_{X}\right)$. Combined with the fact that $K_{X}$ is differentiable at every point of $\operatorname{int} \mathcal{D}\left(K_{X}\right)$ ([2, Corollary 7.1]), this implies that the asserted necessary and sufficient condition for strict convexity of $I_{X}$ is a particular case of [8, Theorem 26.3].

Assume first that $d^{\prime}=d$. In this case $K_{X}$ is strictly convex by [2, Theorem 7.1]; this actually follows immediately from the criterion for equality in Hölder's inequality. Therefore, $K_{X}$ is essentially strictly convex, i.e. $K_{X}$ is strictly convex on every interval contained in the set of points where $K_{X}$ is subdifferentiable. Hence $I_{X}$ is essentially smooth by [8, Theorem 26.3], that is $I_{X}$ is differentiable on the set int $\mathcal{D}\left(I_{X}\right)$, which is required to be non-empty, and $I_{X}$ is steep. By [8, Theorem 26.1], this implies that $I_{X}$ is not subdifferentiable outside of $\operatorname{rint} \mathcal{D}\left(I_{X}\right)$, as required.

In the remaining case $1 \leq d^{\prime} \leq d-1$, put $L:=\operatorname{aff}(\operatorname{supp} X)$. We can assume w.l.o.g. that $0 \in L$, otherwise pick any $\mu \in L$ and use the simple fact that $I_{X}(v)=I_{X-\mu}(v-\mu)$ for $v \in \mathbb{R}^{d}$, which easily implies that our claim holds true for $I_{X}$ if and only if it holds for $I_{X-\mu}$.

Since $L$ is a linear subspace of $\mathbb{R}^{d}$ of dimension $d^{\prime}$, there exists an orthogonal mapping $U: L \rightarrow \mathbb{R}^{d^{\prime}}$. Then by $X \in L$ a.s., for any $v \in L$ we have

$$
I_{X}(v)=\sup _{u \in \mathbb{R}^{d}}\left(u \cdot v-\log \mathbb{E} e^{u \cdot X}\right)=\sup _{u \in L}\left(u \cdot v-\log \mathbb{E} e^{u \cdot X}\right)=I_{U(X)}(U(v)),
$$

where in the last equality we used the change of variables $u \mapsto U(u)$. Therefore, since the mapping $U$ is linear and invertible, $I_{X}$ is subdifferentiable at a $v \in L$ if and only if $I_{U(X)}$ is subdifferentiable at $U(v)$ by [8, Theorem 23.9] (applied with $f=I_{U(X)}$ and $\left.A=U^{-1}\right)$. On the other hand, $v \in \operatorname{rint} \mathcal{D}\left(I_{X}\right)$ if and only if $U(v) \in \operatorname{rint} \mathcal{D}_{U(X)}$, since $U\left(\operatorname{rint} \mathcal{D}\left(I_{X}\right)\right)=\operatorname{rint} U\left(\mathcal{D}\left(I_{X}\right)\right)=\operatorname{rint} \mathcal{D}\left(I_{U(X)}\right)$ by [8, Theorem 6.6]. Thus, by equality (3.4), the case $d^{\prime}<d$ reduces to the case $d^{\prime}=d$ because the support of the random vector $U(X)$ in $\mathbb{R}^{d^{\prime}}$ has full dimension. This finishes the proof of the claim. 
Our proofs of Theorems 1.2 and 2.11 rely on the following technical result, where * stands for convex conjugation (the Legendre-Fenchel transform) of functions on $\mathbb{R}^{d}$.

Lemma 3.1. Let $X$ be a random vector in $\mathbb{R}^{d}, d \geq 1$, and $L$ be a hyperplane in $\mathbb{R}^{d}$ supporting $C_{X}$ and such that $\mathbb{P}(X \in L)>0$. Put $\tilde{K}_{X \mid L}(u):=K_{X \mid L}(u)$ if $u \in \operatorname{Pr}_{L}^{-1}\left(\operatorname{Pr}_{L} \mathcal{D}\left(K_{X}\right)\right)$, otherwise $\tilde{K}_{X \mid L}(u):=+\infty$ for $u \in \mathbb{R}^{d}$. Then

$$
I_{X}(v)=\left(\tilde{K}_{X \mid L}\right)^{*}(v)-\log \mathbb{P}(X \in L), \quad v \in L,
$$

and $\left(\tilde{K}_{X \mid L}\right)^{*}(v)=+\infty$ for $v \notin L$. Moreover, we have

$$
\operatorname{Pr}_{L}\left(\operatorname{rint} \mathcal{D}\left(\tilde{K}_{X \mid L}\right)\right)=\operatorname{Pr}_{L}\left(\operatorname{rint} \mathcal{D}\left(K_{X}\right)\right) .
$$

Proof. Denote by $L_{0}$ the hyperplane passing through 0 that is parallel to $L$, and let $\ell \in \mathbb{R}^{d}$ be the unit vector orthogonal to $L_{0}$ such that $\ell \cdot u \leq \ell \cdot v$ for any $u \in C_{X}$ and $v \in L$. Denote by $\left(v_{1}, v_{2}\right)$ the coordinates of $v \in L$ in $L_{0} \oplus L^{\perp}$, where $L^{\perp}:=\mathbb{R} \ell$.

For any $u_{1} \in L_{0}$ such that $\mathbb{E} e^{\left(u_{1}+u_{2} \ell\right) \cdot X}<\infty$ for some real $u_{2}=u_{2}^{\prime}$, we have

$$
\begin{aligned}
\sup _{u_{2} \in \mathbb{R}}\left(u_{2} v_{2}-\log \mathbb{E} e^{\left(u_{1}+u_{2} \ell\right) \cdot X}\right) & =-\log \left(\inf _{u_{2} \in \mathbb{R}} \mathbb{E} e^{u_{1} \cdot X+u_{2}\left(\ell \cdot X-v_{2}\right)}\right) \\
& =-\log \mathbb{E}\left[e^{u_{1} \cdot X} \mathbb{1}_{\{X \in L\}}\right],
\end{aligned}
$$

where the last equality follows from the dominated convergence theorem applied as $u_{2} \rightarrow+\infty$ using that $e^{u_{1} \cdot X+u_{2}^{\prime}\left(\ell \cdot X-v_{2}\right)}$ is an integrable majorant, which is true because the function $u_{2} \mapsto e^{u_{1} \cdot X+u_{2}\left(\ell \cdot X-v_{2}\right)}$ is non-increasing a.s. by $\ell \cdot X \leq v_{2}$ a.s. On the other hand, if $u_{1} \in L_{0}$ is such that $\mathbb{E} e^{\left(u_{1}+u_{2} \ell\right) \cdot X}=\infty$ for every real $u_{2}$, then the l.h.s. of the first equality in (3.7) is $-\infty$. Therefore,

$$
\begin{aligned}
I_{X}(v) & =\sup _{u_{1} \in L_{0}} \sup _{u_{2} \in \mathbb{R}}\left(u_{1} \cdot v_{1}+u_{2} v_{2}-\log \mathbb{E} e^{\left(u_{1}+u_{2} \ell\right) \cdot X}\right) \\
& =\sup _{\substack{u_{1} \in L_{0}: \\
\left(u_{1}+\mathbb{R} \ell \cap \cap \mathcal{D}\left(K_{X}\right) \neq \varnothing\right.}}\left(u_{1} \cdot v_{1}-\log \mathbb{E}\left[e^{u_{1} \cdot X} \mathbb{1}_{\{X \in L\}}\right]\right) \\
& =\sup _{u_{1} \in \operatorname{Pr}_{L_{0}}\left(\mathcal{D}\left(K_{X}\right)\right)} \sup _{u_{2} \in \mathbb{R}}\left(u_{1} \cdot v_{1}+u_{2} v_{2}-\log \mathbb{E}\left[e^{\left(u_{1}+u_{2} \ell\right) \cdot(X \mid L)}\right]\right)-\log \mathbb{P}(X \in L) \\
& =\sup _{u \in \operatorname{Pr}_{L}^{-1}\left(\operatorname{Pr}_{L} \mathcal{D}\left(K_{X}\right)\right)}\left(u \cdot v-\log \mathbb{E} e^{u \cdot(X \mid L)}\right)-\log \mathbb{P}(X \in L),
\end{aligned}
$$

where in the last equality we used that $L_{0}$ and $L$ are parallel. This proves (3.5).

The claim $\left(\tilde{K}_{X \mid L}\right)^{*}(v)=+\infty$ for $v \notin L$ follows exactly as in (3.1) using that $\tilde{K}_{X \mid L}\left(u_{0}\right)=$ $K_{X \mid L}\left(u_{0}\right)<+\infty$ for any $u_{0} \in L^{\perp}$ by $0 \in \mathcal{D}\left(K_{X}\right)$.

Lastly, it follows from (2.2) that $K_{X \mid L}(u)<+\infty$ when $u \in \operatorname{Pr}_{L}^{-1}\left(\operatorname{Pr}_{L} \mathcal{D}\left(K_{X}\right)\right)$. Therefore, by the definition of $\tilde{K}_{X \mid L}$, we have $\mathcal{D}\left(\tilde{K}_{X \mid L}\right)=\operatorname{Pr}_{L}^{-1}\left(\operatorname{Pr}_{L} \mathcal{D}\left(K_{X}\right)\right)$, and we obtain (3.6) interchanging $\operatorname{Pr}_{L}$ and rint by [8, Theorem 6.6] as follows:

$$
\operatorname{Pr}_{L}\left(\operatorname{rint} \mathcal{D}\left(\tilde{K}_{X \mid L}\right)\right)=\operatorname{rint}\left(\operatorname{Pr}_{L} \mathcal{D}\left(\tilde{K}_{X \mid L}\right)\right)=\operatorname{rint}\left(\operatorname{Pr}_{L} \mathcal{D}\left(K_{X}\right)\right)=\operatorname{Pr}_{L}\left(\operatorname{rint} \mathcal{D}\left(K_{X}\right)\right) .
$$

Proof of Theorem 1.2. Let $L$ be a hyperplane supporting $C_{X}$ and such that $\mathbb{P}(X \in L)>0$. By Lemma 3.1 and Proposition 1.1.a applied to $X \mid L$, we have $I_{X \mid L}(v)=\left(K_{X \mid L}\right)^{*}(v)=+\infty$ and $\left(\tilde{K}_{X \mid L}\right)^{*}(v)=+\infty$ for $v \notin L$. Therefore, the functions $\left(\tilde{K}_{X \mid L}\right)^{*}$ and $\left(K_{X \mid L}\right)^{*}$ coincide if they are equal on $L$. Thus, (3.5) implies that

$$
I_{X}(v)=I_{X \mid L}(v)-\log \mathbb{P}(X \in L), \quad v \in L,
$$

if and only if $\left(\tilde{K}_{X \mid L}\right)^{*}=\left(K_{X \mid L}\right)^{*}$. This is in turn equivalent to $\left(\tilde{K}_{X \mid L}\right)^{* *}=K_{X \mid L}$ (by [8, Theorem 12.2]) because $\tilde{K}_{X \mid L}$ is a convex function (this follows from the definition of $\tilde{K}_{X \mid L}$ ) and $K_{X \mid L}$ is a lower semi-continuous convex function (by [2, Theorem 7.1]), both 
When is the rate function of a random vector strictly convex?

finite at least at one point. The last equality holds true if and only if $\tilde{K}_{X \mid L}$ equals $K_{X \mid L}$ except possibly at some relative boundary points of $\mathcal{D}\left(\tilde{K}_{X \mid L}\right)$ (by [8, Theorem 7.4]). Thus, equalities (1.2) and $\operatorname{rint} \mathcal{D}\left(\tilde{K}_{X \mid L}\right)=\operatorname{rint} \mathcal{D}\left(K_{X \mid L}\right)$ are equivalent.

The latter one is equivalent to $\operatorname{Pr}_{L}\left(\operatorname{rint} \mathcal{D}\left(\tilde{K}_{X \mid L}\right)\right)=\operatorname{Pr}_{L}\left(\operatorname{rint} \mathcal{D}\left(K_{X \mid L}\right)\right)$ because the sets $\operatorname{rint} \mathcal{D}\left(\tilde{K}_{X \mid L}\right)$ and $\operatorname{rint} \mathcal{D}\left(K_{X \mid L}\right)$ are right cylinders by [8, Corollary 6.6.2]. Hence, by (3.6), equalities (1.2) and (1.3) are equivalent, as claimed.

Proof of Theorem 2.11. a) Let us prove the first inclusion in (2.4). Let $F \in \mathcal{F}^{*}\left(\mathcal{D}\left(I_{X}\right)\right)$ be a maximal proper face of $\mathcal{D}\left(I_{X}\right)$. Then there is a hyperplane $L$ supporting the convex set $\mathcal{D}\left(I_{X}\right)$ such that $F=\mathcal{D}\left(I_{X}\right) \cap L$. The hyperplane $L$ also supports $\operatorname{cl} C_{X}$ by the second inclusion in (1.1). Moreover, we have $\mathbb{P}(X \in L)>0$ since otherwise $\mathcal{D}\left(I_{X}\right) \cap L=\varnothing$ by Proposition 1.1.a, which is a contradiction. Therefore, $L \cap C_{X} \neq \varnothing$, and thus $L$ supports $C_{X}$. Hence $C:=C_{X} \cap L$ is a face of $C_{X}$. We also have $F=\mathcal{D}\left(I_{X \mid C}\right)$ by (1.2) and the fact that $X \mid C$ has the same distribution as $X \mid L$ (as $\mathbb{P}(X \in L \backslash C)=0$ ). Hence $F=\mathcal{D}\left(I_{X}\right) \cap \operatorname{cl} C_{X \mid C}$ by (1.1).

Clearly, $C$ is a proper face of $C_{X}$ (i.e. $C \neq C_{X}$ ) since otherwise $F$ cannot be a proper face of $\mathcal{D}\left(I_{X}\right)$. However, $C$ is not necessarily a maximal proper face. Let $C^{\prime} \in \mathcal{F}^{*}\left(C_{X}\right)$ be such that $C \subset C^{\prime}$. Since this is an exposed face of $C_{X}$, there is a hyperplane $L^{\prime}$ supporting $C_{X}$ and satisfying $C^{\prime}=C_{X} \cap L^{\prime}$. We have

$$
\mathbb{P}\left(X \in L^{\prime}\right)=\mathbb{P}\left(X \in C^{\prime}\right) \geq \mathbb{P}(X \in C)>0 .
$$

Since $L^{\prime}$ supports $C_{X}$, equality (1.2) is valid with $L=L^{\prime}$ and it implies that the set $F^{\prime}:=\mathcal{D}\left(I_{X}\right) \cap L^{\prime}$ satisfies $F^{\prime}=\mathcal{D}\left(I_{X \mid C^{\prime}}\right)$ and therefore is non-empty; moreover, we have $F^{\prime}=\mathcal{D}\left(I_{X}\right) \cap \operatorname{cl} C_{X \mid C^{\prime}}$ by (1.1). This shows that $F^{\prime}$ is a proper face of $\mathcal{D}\left(I_{X}\right)$ since $L^{\prime}$ supports $\mathcal{D}\left(I_{X}\right)$ by (1.1).

Finally, by $C \subset C^{\prime}$, we have $C_{X \mid C} \subset C_{X \mid C^{\prime}}$, and thus

$$
F=\mathcal{D}\left(I_{X}\right) \cap \operatorname{cl} C_{X \mid C} \subset \mathcal{D}\left(I_{X}\right) \cap \operatorname{cl} C_{X \mid C^{\prime}}=F^{\prime} .
$$

Therefore, $F=F^{\prime}$ since $F$ is a maximal proper face by the assumption. Thus, we have $F=\mathcal{D}\left(I_{X \mid C^{\prime}}\right)$, which proves the first inclusion in (2.4).

To prove the remaining inclusion in (2.4), pick a $C^{\prime} \in \mathcal{F}_{+}^{*}\left(C_{X}\right)$. Then $C^{\prime}=C_{X} \cap L^{\prime}$ for some hyperplane $L^{\prime}$ supporting $C_{X}$ and satisfying $\mathbb{P}\left(X \in L^{\prime}\right)>0$. As we have shown just above, $F^{\prime}:=\mathcal{D}\left(I_{X}\right) \cap L^{\prime}$ is a non-empty proper face of $\mathcal{D}\left(I_{X}\right)$ (but it is not necessarily a maximal one anymore) and $F^{\prime}=\mathcal{D}\left(I_{X \mid C^{\prime}}\right)$. This finishes the proof of Part a).

b) Direct implication. Assume that $I_{X}$ is strictly convex. Let us use induction in $\operatorname{dim} C_{X}$ to prove that int $\mathcal{D}\left(K_{X}\right) \neq \varnothing, K_{X}$ has the projection property, and $K_{X}$ is totally steep.

This claim holds trivially in the base case $\operatorname{dim} C_{X}=0$, where $X$ is constant a.s.

To prove the induction step, consider any hyperplane $L$ supporting $C_{X}$ such that $0<\mathbb{P}(X \in L)<1$. By Lemma 3.1, the effective domain of the function $\left(\tilde{K}_{X \mid L}\right)^{*}$ is contained in $L$. Therefore, this function is strictly convex by (3.5) because $I_{X}$ is strictly convex by the assumption. Then $\operatorname{int} \mathcal{D}\left(\left(\tilde{K}_{X \mid L}\right)^{* *}\right) \neq \varnothing$ and $\left(\tilde{K}_{X \mid L}\right)^{* *}$ is steep by [8, Theorem 26.3] (because strict convexity implies essential strict convexity). Hence $\operatorname{int} \mathcal{D}\left(\tilde{K}_{X \mid L}\right) \neq \varnothing$ and $\tilde{K}_{X \mid L}$ is also steep because it equals $\left(\tilde{K}_{X \mid L}\right)^{* *}$ except possibly at some relative boundary points of $\mathcal{D}\left(\tilde{K}_{X \mid L}\right)$ ([8, Theorems 7.4 and 12.2]).

Let us show that $\operatorname{int} \mathcal{D}\left(\tilde{K}_{X \mid L}\right)=\operatorname{int} \mathcal{D}\left(K_{X \mid L}\right)$. Otherwise, by $\mathcal{D}\left(\tilde{K}_{X \mid L}\right) \subset \mathcal{D}\left(K_{X \mid L}\right)$ and convexity of $\mathcal{D}\left(K_{X \mid L}\right)$, there is a point $u \in \partial \mathcal{D}\left(\tilde{K}_{X \mid L}\right) \cap \operatorname{int} \mathcal{D}\left(K_{X \mid L}\right)$. Pick a sequence $u_{1}, u_{2}, \ldots$ in $\operatorname{int} \mathcal{D}\left(\tilde{K}_{X \mid L}\right)$ converging to $u$. Then $\lim _{n \rightarrow \infty}\left|\nabla \tilde{K}_{X \mid L}\left(u_{n}\right)\right|=\infty$ by steepness of $\tilde{K}_{X \mid L}$. Thus, $\lim _{n \rightarrow \infty}\left|\nabla K_{X \mid L}\left(u_{n}\right)\right|=\infty$ because $\tilde{K}_{X \mid L}$ equals $K_{X \mid L}$ whenever $\tilde{K}_{X \mid L}<\infty$, and hence $\tilde{K}_{X \mid L}=K_{X \mid L}$ on $\operatorname{int} \mathcal{D}\left(\tilde{K}_{X \mid L}\right)$. However, it must be $\lim _{n \rightarrow \infty}\left|\nabla K_{X \mid L}\left(u_{n}\right)\right|=$ 
When is the rate function of a random vector strictly convex?

$\left|\nabla K_{X \mid L}(u)\right|$ because $K_{X \mid L}$ is continuously differentiable on $\operatorname{int} \mathcal{D}\left(K_{X \mid L}\right)$ since so is the Laplace transform of any random variable ([2, Corollary 7.1]). This is a contradiction.

We now have $\operatorname{Pr}_{L}\left(\operatorname{int} \mathcal{D}\left(\tilde{K}_{X \mid L}\right)\right)=\operatorname{Pr}_{L}\left(\operatorname{int} \mathcal{D}\left(K_{X \mid L}\right)\right)$, which implies equality (1.3) by (3.6). Thus, $K_{X}$ satisfies Condition a) in Definition 2.5 of the projection property because $L$ was chosen arbitrarily. Equality (1.3) in turn implies (1.2) by Theorem 1.2, hence $I_{X \mid L}$ is strictly convex because so is $I_{X}$ and $\mathcal{D}\left(I_{X \mid L}\right) \subset L$ by Proposition 1.1.a.

For any maximal proper face $C \in \mathcal{F}_{+}^{*}\left(C_{X}\right)$, pick a hyperplane $L$ supporting $C_{X}$ such that $C=C_{X} \cap L$. Since $0<\mathbb{P}(X \in L)<1$ and $\operatorname{dim}(\operatorname{supp}(X \mid L))<\operatorname{dim}(\operatorname{supp} X)$, we can apply the assumption of induction to the random vector $X \mid L$, which is distributed as $X \mid C$ and has strictly convex rate function $I_{X \mid L}$ by the above. Therefore, $\operatorname{int} \mathcal{D}\left(K_{X \mid C}\right) \neq \varnothing$, $K_{X \mid C}$ has the projection property, and $K_{X \mid C}$ is totally steep. Thus, since $C$ was chosen arbitrarily, $K_{X}$ has the projection property, as required. Finally, $K_{X}$ is totally steep, as required, since $\operatorname{int} \mathcal{D}\left(K_{X}\right) \neq \varnothing$ and $K_{X}$ is steep by Proposition 1.1.b.

Reverse implication. Assume that $\varnothing \neq \operatorname{int} \mathcal{D}\left(K_{X}\right), K_{X}$ has the projection property, and $K_{X}$ is totally steep. We again use induction in $\operatorname{dim} C_{X}$ to show that $I_{X}$ is strictly convex.

In the base case $\operatorname{dim} C_{X}=0$, the set $\mathcal{D}\left(I_{X}\right)$ consists of a single point, and the claim holds vacuously.

To prove the induction step, pick a closed line segment $J \subset \mathcal{D}\left(I_{X}\right)$. From (2.1) and the definition of a face, either $\operatorname{rint} J \subset \operatorname{rint} \mathcal{D}\left(I_{X}\right)$ or $J$ is contained in some maximal proper face of $\mathcal{D}\left(I_{X}\right)$. In the former case, $I_{X}$ is not affine on $J$ by Proposition 1.1.b. In the latter case, by the first inclusion in (2.4), we have $J \subset \mathcal{D}\left(I_{X \mid C}\right) \subset C$ for some face $C \in \mathcal{F}_{+}^{*}\left(C_{X}\right)$. Note that $\varnothing \neq \operatorname{int} \mathcal{D}\left(K_{X}\right) \subset \operatorname{int} \mathcal{D}\left(K_{X \mid C}\right)$ (cf. (2.2)), $K_{X \mid C}$ is totally steep since so is $K_{X}$, and $K_{X \mid C}$ has the projection property since $K_{X}$ has this property. Therefore, $I_{X \mid C}$ is strictly convex by $\operatorname{dim}(\operatorname{supp}(X \mid C))<\operatorname{dim}(\operatorname{supp} X)$ and the assumption of induction. Hence $I_{X}$ is not affine on $J$ because for some hyperplane $L$ supporting $C_{X}$ and such that $C=C_{X} \cap L$, we have $I_{X}=I_{X \mid L}=I_{X \mid C}$ by (1.2), which holds true by Theorem 1.2 because $K_{X}$ has the projection property. Therefore, $I_{X}$ is not affine on $J$ in either case and thus $I_{X}$ is strictly convex.

\section{References}

[1] Akopyan, A. and Vysotsky, V.: Large deviations of convex hulls of planar random walks and Brownian motions. Accepted in Ann. H. Lebesgue, (2021). arXiv:1606.07141v4

[2] Barndorff-Nielsen, O.: Information and exponential families in statistical theory. Reprint of the 1978 original. John Wiley \& Sons, Ltd., Chichester, 2014. MR-3221776

[3] Bahadur, R.R. and Zabell, S.L.: Large deviations of the sample mean in general vector spaces. Ann. Probab. 7, (1979), 587-621. MR-0537209

[4] Dembo, A. and Zeitouni, O.: Large Deviations Techniques and Applications. Corrected reprint of the second edition. Springer-Verlag, Berlin, 2010. MR-2571413

[5] Lifshits, M.A.: Large deviation principle: processes and empirical distributions (in Russian). St. Petersburg State University, St. Petersburg, 2002. https://sites.google.com/site/ mlprobability/home10/ml09/Mall4.pdf

[6] Mogulskii, A.A.: Large deviations for processes with independent increments. Ann. Probab. 21, (1993), 202-215. MR-1207223

[7] Rassoul-Agha, F. and Seppäläinen, T.: A Course on Large Deviations with an Introduction to Gibbs Measures. American Mathematical Society, Providence, RI, 2015. MR-3309619

[8] Rockafellar, R.T.: Convex Analysis. Princeton University Press, Princeton, NJ, 1970. MR0274683

[9] Widder, D.V.: The Laplace Transform. Princeton University Press, Princeton, NJ, 1941. MR0005923

Acknowledgments. I thank the anonymous referee for comments and suggestions. 


\section{Electronic Journal of Probability Electronic Communications in Probability}

\section{Advantages of publishing in EJP-ECP}

- Very high standards

- Free for authors, free for readers

- Quick publication (no backlog)

- Secure publication $\left(\mathrm{LOCKSS}^{1}\right)$

- Easy interface (EJMS²)

\section{Economical model of EJP-ECP}

- Non profit, sponsored by $\mathrm{IMS}^{3}, \mathrm{BS}^{4}$, ProjectEuclid ${ }^{5}$

- Purely electronic

\section{Help keep the journal free and vigorous}

- Donate to the IMS open access fund ${ }^{6}$ (click here to donate!)

- Submit your best articles to EJP-ECP

- Choose EJP-ECP over for-profit journals

\footnotetext{
${ }^{1}$ LOCKSS: Lots of Copies Keep Stuff Safe http://www. lockss.org/

${ }^{2}$ EJMS: Electronic Journal Management System http://www.vtex.lt/en/ejms.html

${ }^{3}$ IMS: Institute of Mathematical Statistics http://www.imstat.org/

${ }^{4}$ BS: Bernoulli Society http://www. bernoulli-society.org/

${ }^{5}$ Project Euclid: https://projecteuclid.org/

${ }^{6}$ IMS Open Access Fund: http://www.imstat.org/publications/open.htm
} 